\title{
PROTOSTELLAR COLLAPSE WITH A SHOCK
}

\author{
JoHN C. TSAI \\ NASA/Ames Research Center, Mail Stop 245-3, Moffett Field, CA 94035-1000 \\ AND \\ Juliana J. L. Hsu \\ Theoretical Astrophysics Center, University of California, Berkeley, Berkeley, CA 94720 \\ Received 1994 November 10; accepted 1995 February 7
}

\begin{abstract}
We reexamine both numerically and analytically the collapse of the singular isothermal sphere in the context of low-mass star formation. We consider the case where the onset of collapse is initiated by some arbitrary process which is accompanied by a central output of either heat or kinetic energy. We find two classes of numerical solutions describing this manner of collapse. The first approaches in time the expansion wave solution of Shu, while the second class is characterized by an ever-decreasing central accretion rate and the presence of an outwardly propagating weak shock. The collapse solution which represents the dividing case between these two classes is determined analytically by a similarity analysis. This solution shares with the expansion wave solution the properties that the gas remains stationary with an $r^{-2}$ density profile at large radius and that, at small radius, the gas free-falls onto a nascent core at a constant rate which depends only on the isothermal sound speed. This accretion rate is a factor of $\sim 0.1$ that predicted by the expansion wave solution. This reduction is due in part to the presence of a weak shock which propagates outward at 1.26 times the sound speed. Gas in the postshock region first moves out subsonically but is then decelerated and begins to collapse. The existence of two classes of numerical collapse solutions is explained in terms of the instability to radial perturbations of the analytic solution. Collapse occurring in the manner described by some of our solutions would eventually unbind a finite-sized core. However, this does not constitute a violation of the instability properties of the singular isothermal sphere which is unstable both to collapse and to expansion. To emphasize this, we consider a purely expanding solution for isothermal spheres. This solution is found to be self-similar and results in a uniform density core in the central regions of the gas. Our solutions may be relevant to the "luminosity" problem of protostellar cores since the predicted central accretion rates are significantly reduced relative to that of the expansion wave solution. Furthermore, our calculations indicate that star-forming cloud cores are not very tightly bound and that modest disturbances can easily result in both termination of infall and dispersal of unaccreted material.
\end{abstract}

Subject headings: hydrodynamics - shock waves - stars: formation

\section{INTRODUCTION}

The gravitational collapse of gas spheres in the context of star formation has long been an active area of study. Pioneering numerical calculations (e.g., Gaustad 1963; Bodenheimer \& Sweigart 1968; Larson 1969; Penston 1969a, b) based on the collapse of a Jeans unstable uniform density sphere elucidated the general properties of star formation and provided the basis of much of the subsequent work in the area. One of the most important properties found by these early investigations was that efficient cooling by dust grains allowed the rapid radiation of compressional heat generated during collapse. The gas remained essentially isothermal with a temperature of $\sim 10 \mathrm{~K}$ over many orders of magnitude in density during the early phases of collapse. Isothermality was only violated when the densities became sufficiently high for gas to become rather optically thick to dust emission. Isothermality, taken as an assumption, enabled great simplification in many subsequent studies because radiation transfer was eliminated from the problem (however, this restricted the applicability of these solutions to the more optically thin regions of collapse). It was also shown that collapse occurred in a very nonhomologous fashion, i.e., the central regions of the cloud collapsed much more rapidly than the outer regions. The density distribution inevitably approached a strongly peaked $r^{-2}$ profile as the collapse approached core formation.

Later studies addressed primarily the unrealistic nature of the starting conditions adopted in the early work. The assumed initial configuration for the gas was an isothermal uniform density sphere that was far out of hydrostatic equilibrium. Such conditions would be very difficult to produce in nature. Furthermore, the velocities resulting from the collapse were criticized as being unrealistic (Shu 1977). At large radius, the flow was directed inward at 3.3 times the sound speed which was considered numerically ad hoc. Many of the subsequent considerations (e.g., Shu 1977; Hunter 1977; Boss \& Black 1982; Whitworth \& Summers 1985; Foster \& Chevalier 1993) centered around the collapse of equilibrium isothermal spheres (Bonner-Ebert spheres; see Ebert 1955; Bonner 1956) where the gas was assumed to be isothermal at all times and initially stationary. Because the initial configurations were in equilibrium, the numerical calculations of collapse were initiated by some rather ad hoc perturbation. However, the nature of the collapse was found to be rather insensitive to the manner of the initial perturbation (e.g., Hunter 1977).

Many collapse solutions were found. These differed from one another based on the initially assumed density profile and 

ranged from the collapse of the marginally unstable BonnerEbert sphere (Foster \& Chevalier 1993) to the maximally unstable singular isothermal sphere (Shu 1977; Hunter 1977). However, it was not clear which, if any, was the most relevant to present-day low-mass star formation. Recently, both theoretical and observational work has yielded a paradigm for lowmass star formation (see Shu et al. 1993 and references therein). A cloud initially supported against collapse by gas pressure, turbulence, and magnetic fields evolves quasistatically toward a centrally condensed singular isothermal sphere due to the outward diffusion of the magnetic field (ambipolar diffusion). After $\sim 10^{6} \mathrm{yr}$, the distribution becomes sufficiently centrally condensed to undergo an "inside-out" collapse in the manner of the analytic, self-similar solution of Shu (1977) (dubbed the “expansion wave solution," hereafter EWS). Although the collapse calculation has been generalized to account for modest amounts of rotation (Terebey, Shu, \& Cassen 1984) and magnetic fields (Galli \& Shu 1993a, b), the simplicity of the original solution, as well as its closeness to the generalized solutions at relatively early times and in regions somewhat outside the core, has led it to be generally adopted as the benchmark for testing the agreement of the paradigm with observations.

The EWS is obtained when a static singular isothermal sphere suffers a central perturbation which causes the core to collapse. The ensuing collapse proceeds in an "inside-out" fashion where the infalling region is bounded by a rarefaction wave which propagates outward at the sound speed. Outside the rarefaction wave, the gas remains undisturbed and in hydrostatic equilibrium. At small radius, gas free-falls onto a growing core at a constant rate which depends only on the sound speed $a$ and the gravitational constant $G$,

$$
\dot{M}=C \frac{a^{3}}{G},
$$

where $C=0.975$ for the EWS. The collapse can be initiated by any process which causes an unbalancing of the two forces in the core (Shu 1977) or by the onset of true dynamical instability (Shu, Adams, \& Lizano 1987). In the former case, anything which decreases the central gas pressure, increases the gravity (mass), or both, will cause core collapse. Possible physical processes include molecule formation (Shu 1977) and primary core collapse (e.g., Larson 1969; Winkler \& Newman 1980). True dynamical instability arises during the evolution of the protostellar cloud toward ever more centrally peaked density profiles during the process of ambipolar diffusion.

The considerations of this paper derive from the observation that the suggested processes which might initiate the EWS collapse will not only cause an initial unbalancing of the opposing central forces, but will also in general be accompanied by some initial or sustained burst of energy which will be reabsorbed by parts of the collapsing cloud. Certainly, immediately after the onset of collapse in the inside-out picture, energy will be released from a radiating accretion shock around either a core or a disk, or possibly by gas streams, which are not flowing exactly radially, colliding outside of the core. This energy will propagate back into static and subsonically moving parts of the envelope and cause a preheating effect. That is, the radiation will heat the gas above the assumed isothermal temperature, causing an outward push due to a higher gas pressure. This extra pressure may have significant dynamical effects. Other ways in which collapse can be initiated may also be accompanied by energetic output, such as molecule formation which produces heat via recombination. Additionally, sustained stellar winds produced in the very early life of a nascent protostar could push material out via kinetic pressure. In any case, since the conditions which initiate protostellar collapse are poorly understood, it is important to investigate a spectrum of possibilities, the results of which may be compared to observations in order to better understand star formation.

We can imagine intuitively what the effects might be of absorption of an initial burst of energy followed by an unbalancing of the pressure in the core of a static, isothermal sphere. The extra energy or pressure will first cause an outward push to the gas in the central regions. In one instance, if the amount of energy or the outward push is very small, the initial condition may be thought of as a perturbation on the condition which gives rise to the EWS. We might imagine that in time, as the ensuing collapse progresses to encompass scales much larger than that set by the initial condition, the effects of the perturbation will die out, and the EWS will be recovered. In another case, if the outward push is stronger, a shock will likely form. Because of the steep density gradient of the singular isothermal sphere, this shock can coast to large radius. In the center, after the initial burst has subsided, gas which was pushed outward will decelerate due to the pressure imbalance, eventually going into infall.

We numerically determine collapse solutions resulting from the energy burst initial condition in $\S 2$. However, in the case where a shock forms, we might expect in analogy with the Sedov-Taylor solution (e.g., Sedov 1959) that there would be an evolution toward a similarity solution once the shock has moved to distances much greater than the scale set by the initial perturbation. Recall that the Sedov-Taylor solution describes the state of gas, for example, after a piston pushes into an initially static uniform density gas (gravity is not considered). The motion of the piston gives rise to a shock. When the shock has propagated into a region far removed from the scale set by the motion of the piston, the solution approaches self-similarity because the initial length scale becomes irrelevant. By analogy, when the shock in our problem has moved beyond the core area where the initial energy burst occurred, there are also no longer any relevant length scales. Contrary to this view, we find that the numerically generated solutions do not tend toward any similiarity solution; rather, they depend on the particular conditions initiating the collapse. However, this result can be understood in terms of the instability of a self-similar solution which we present in $\S 3$. In $\S 4$, we introduce an expanding solution to help elucidate the properties of the new collapse solutions as well as the general collapse properties of the singular isothermal sphere. We discuss the relation of the new solutions to the EWS and the theory of star formation in $\S 5$. Conclusions are also given in $\S 5$.

\section{NUMERICAL SOLUTION}

\subsection{Method}

The case where radiation from an accretion front preheats gas which is not yet in a state of free fall is best treated by a hydrodynamical code with radiation transfer. Although similar calculations of collapse have been carried out (e.g., Morfill, Tscharnuter, \& Völk 1985; Bodenheimer et al. 1990; Yorke, Bodenheimer, \& Laughlin 1993), the initial conditions were always assumed to be somewhat out of hydrostatic equi- 
librium. We will report elsewhere on a one-dimensional calculation treating the effects of accretion luminosity assuming the exact singular isothermal sphere as the initial state. In the present paper, we take a less specific approach by approximating the effects of the energy deposition by a piston. Although this approach is more heuristic than solving a radiative transfer problem, it lends itself to a general elucidation of the relevant physics of the problem and the consideration of other possible modes of energy deposition in the center of the cloud.

We assume that the protostellar cloud is spherically symmetric, is always isothermal, and obeys the ideal gas equation of state. The dynamical calculations are carried out with a Lagrangian hydrodynamics code modified from that developed by Yahil, Johnston, \& Burrows (1987). The code is conservative in the fluxes of energy and momentum and has second-order spatial accuracy in the fluxes.

The initial configuration of the cloud is a singular isothermal sphere. We begin a calculation by assuming that the material within a radius of $2 \times 10^{13} \mathrm{~cm}$, which has an enclosed mass of $2.5 \times 10^{-4} M_{\odot}$ for a cloud temperature of $20 \mathrm{~K}$ and a mean molecular weight $\mu$ of 2 , forms a protostellar core. If there is no energy input, and the material immediately outside of this region is forced into free fall, we recover the EWS.

A major problem with using a Lagrangian code for an accretion calculation is that the Courant time can be so reduced for the region near the central object that it makes the investigation of the long-term evolution of the rest of the object extremely difficult. We circumvent this problem by specifying a critical radius such that if a shell of material (corresponding to a zone in the calculation) falls below this critical radius, it is assumed to have been accreted onto the protostellar core, which then only acts gravitationally on the rest of the cloud. The mass accretion rate can then be approximated by $m_{\text {shell }} / \Delta t$, where $m_{\text {shell }}$ is the mass of the shell and $\Delta t$ is the time elapsed since the last shell was accreted. This accretion rate is then held constant until the next shell is accreted. The accretion rate calculated this way for the EWS follows the analytical value closely, with $<1 \%$ variations, due to changes of zone sizes and time steps.

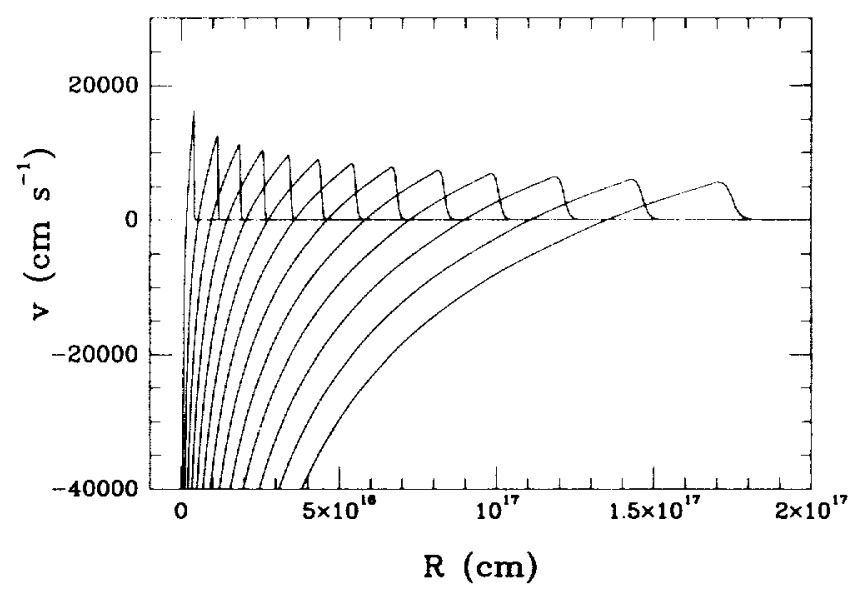

Fig. $1 a$
We simulate the input of the initial burst of energy by applying a piston with a velocity $v_{p}$ immediately outside the protostellar core and starting at the critical radius of $2 \times 10^{13} \mathrm{~cm}$. The piston is pushed at a constant velocity until it has reached some distance $d_{p}$, where the piston is instantaneously stopped and we apply the free-fall boundary condition. This boundary condition corresponds to the limiting case where the inner pressure is reduced to zero in order to initiate collapse. Tests with different boundary conditions where the piston is allowed to decelerate gradually under the influence of less severe pressure gradients give similar results, so they are not discussed. We assume a constant pressure outer boundary condition.

\subsection{Results}

In the first case considered (case [i]), we move the piston at half the isothermal sound speed $\left(a=2.87 \times 10^{4} \mathrm{~cm} \mathrm{~s}^{-1}\right.$ for our chosen conditions) to a distance of $d_{p}=2 \times 10^{14} \mathrm{~cm}$. The resulting velocity and density profiles of the gas at different times are plotted in Figures $1 a$ and $2 a$, respectively, and the accretion rate at the core radius is given by the uppermost solid curve of Figure 3. The action of the piston forms a shock which propagates outward. Despite the subsonic motion of the piston, a shock is formed because the buildup of material in front of the piston causes a supersonically moving front to advance ahead of the piston. The material outside of the shock remains stationary with a density given by that of the singular isothermal sphere. Inside the shock, gas is flowing outward subsonically, having been accelerated by the passage of the shock. The gas eventually is decelerated by the pressure deficit at small radius and goes into free fall at the inner boundary. The strength of the shock declines with time and eventually becomes an acoustic wave propagating outward at the sound speed. The region of outflowing gas and the outward velocity of postshock gas become correspondingly smaller as the shock degrades. Similarly, the size of the discontinuity in the density profile decreases with time. The accretion rate starts out low (after settling down from transients) but after a very short time rises rapidly and tends toward the EWS value appropriate to the chosen temperature of $20 \mathrm{~K}$.

This case corresponds to a collapse which is initially per-

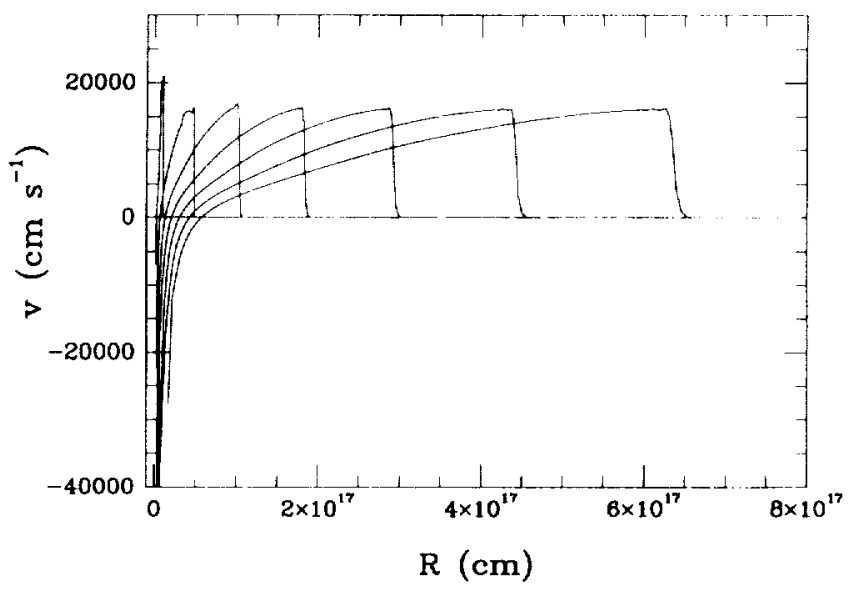

FIG. $1 b$

Fig. 1. (a) The velocity profiles of the gas for the case where $v_{p}=0.5 a$ and $d_{p}=2 \times 10^{14} \mathrm{~cm}$ are plotted at several different times. The leftmost curve corresponds to the earliest time of $0.1 \times 10^{12} \mathrm{~s}$ after starting the collapse. Moving toward the right, the curves correspond to times of (in units of $10^{12} \mathrm{~s}$ ), $0.307,0.507$, $0.723,0.967,1.25,1.57,1.97,2.42,2.95,3.58,4.35$, and 5.24 . (b) The velocity profiles of the gas for the case where $v_{p}=0.5 a$ and $d_{p}=2 \times 10^{15} \mathrm{~cm}$ are plotted at times (in units of $10^{12}$ s) $0.239,1.18,2.63,4.74,7.6,11.6$, and 16.7. The leftmost curve gives the earliest time, and the rightmost curve gives the latest time. 


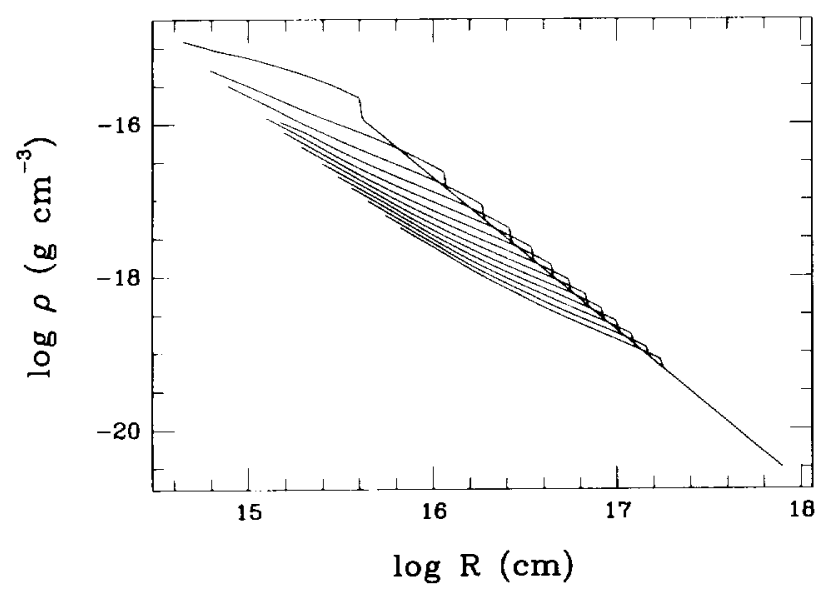

FIG. $2 a$

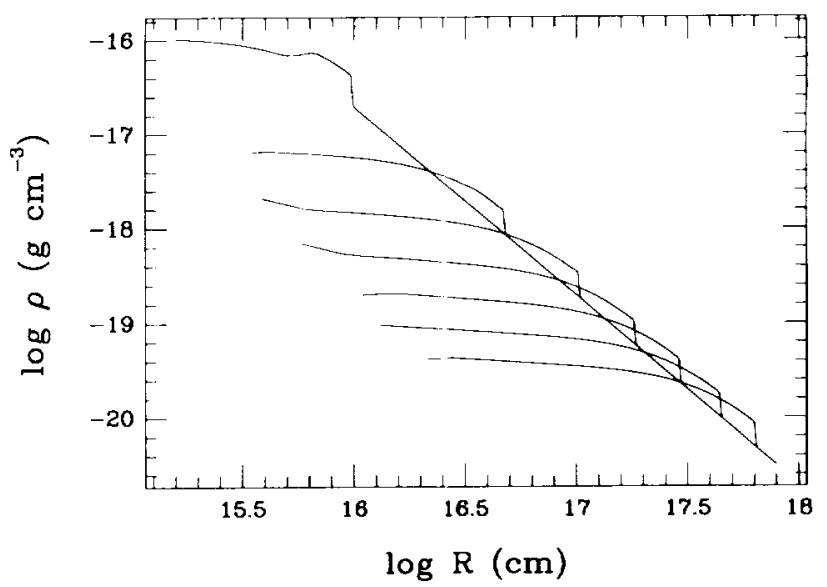

FIG. 2b

Fig. 2. - (a) The density profiles of the gas for the case where $v_{p}=0.5 a$ and $d_{p}=2 \times 10^{14} \mathrm{~cm}$ are plotted for the same set of times as Fig. 1a. The topmost curve corresponds to the earliest time of $0.1 \times 10^{12} \mathrm{~s}$, and successively lower curves correspond to later times. $(b)$ The density profiles of the gas for the case where $v_{p}=0.5 a$ and $d_{p}=2 \times 10^{15} \mathrm{~cm}$ are plotted for the same set of times as Fig. 1b. The topmost curve gives the earliest time, and lower curves give later times.

turbed from the EWS by the action of the piston. (Recall that we obtain the EWS by simply unbalancing the forces in the center with no accompanying piston moving outward.) However, the effects of the perturbation die out rapidly, and the collapse approaches the EWS solution.

On pushing the piston out farther to $d_{p}=2 \times 10^{15} \mathrm{~cm}$ with the same velocity $v_{p}$ (case [ii]), we obtain the gas velocity and density profiles shown in Figures $1 b$ and $2 b$, respectively, and an accretion rate given by the lowest solid curve of Figure 3 . We again get a shock formed by the early action of the piston, but the shock persists in time with constant amplitude all the way to the outer boundary. As before, the gas is stationary outside the shock and outflowing immediately inside of it. However, because of the undiminished amplitude of the shock, the outflowing gas occupies a significant fraction of the nonstatic region. The shock is rather weak with a Mach number of $\sim 1.26$, and the gas has a postshock outflow velocity

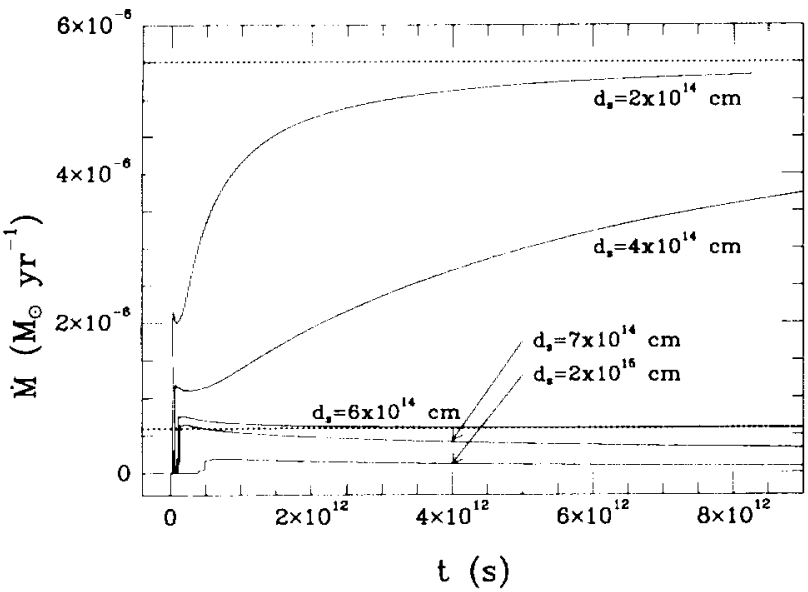

Fig. 3.-The central mass accretion rates for several cases are plotted vs. time. All cases shown (solid lines) assume $v_{p}=0.5 a$ but have different values of $d_{p}$. Starting from the topmost curve and going toward the lowest curve, the assumed values of $d$ are $2 \times 10^{14} \mathrm{~cm}, 4 \times 10^{14} \mathrm{~cm}, 6 \times 10^{14} \mathrm{~cm}, 7 \times 10^{14} \mathrm{~cm}$, and $2 \times 10^{15} \mathrm{~cm}$. The upper dotted line gives the constant mass accretion rate of the EWS (see eq. [1]), and the lower dotted line gives the constant rate of the shock solution. of $\sim 0.43 \mathrm{a}$. The postshock density rises above the value given by the singular isothermal sphere for a considerable region inside the shock. The central mass accretion rate starts out very low and declines with time and never rises back up to the EWS value. In pushing the piston out to larger distances than in case (i), we have essentially perturbed the collapse farther away from the EWS solution. We might have expected that the net effect would be for the collapse to take a much longer time than that of case (i) to evolve back to the EWS. Instead, the collapse does not show a tendency to evolve back to the EWS at all.

Several cases with values of $d_{p}$ between those of cases (i) and (ii) above are also computed ( $v_{p}=0.5 a$ in all these cases). The central accretion rates for these collapses are shown in Figure 3 . The cases where the central accretion rate increases with time have gas velocity and density profiles which behave in time similar to those of case (i), and cases with declining accretion rates have velocities and densities similar to those of case (ii). We see that two kinds of solutions are obtained from the piston experiments. When the collapse is initiated by a piston which pushes to something less than $\sim 6 \times 10^{14} \mathrm{~cm}$, the nature of the subsequent flow is to evolve secularly back toward the EWS. For simplicity we call these class I solutions. Once the piston is allowed to push farther out than this, the evolution of the flow is toward ever decreasing accretion rates (class II solutions). The farther out the piston is allowed to go, the smaller is the accretion rate.

In Figure 4, we show the mass of the inner sink cell (the "protostar") as a function of time for the cases shown in Figure 3. The times required to accrete a sizable mass $\left(\sim 1 M_{\odot}\right)$ onto the sink cell can be significantly extended relative to the dynamical time if the collapse proceeds according to the lower curves of Figure 4 . In these cases, the mass of the sink cell would only be $\sim 0.1 M_{\odot}$ by the time the shock hits the boundary of the calculation. If star formation were to occur in this manner, the accretion of a large fraction of the mass of the protostar occurs after the passage of the shock through the boundary, assuming a typical size for the cloud core. The subsequent collapse would then depend on the particular properties of this boundary.

We obtain the same set of results as above for different assumed piston speeds. We do not show the gas velocities and 


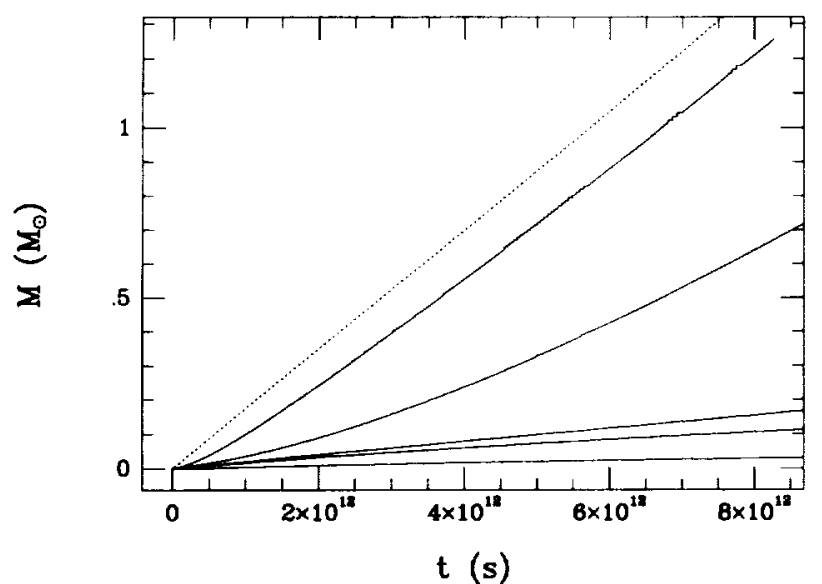

Fig. 4.-The mass of the central star is plotted as a function of time for the same cases as shown in Fig. 3. The top curve corresponds to the case where $d_{p}=2 \times 10^{14} \mathrm{~cm}$, the next highest curve gives the case where $d_{p}=4 \times 10^{14}$ $\mathrm{cm}$, and so forth for the lower curves. The dotted line gives the mass for the EWS solution.

densities in detail. Figure 5 shows the mass accretion rates for some of the cases considered. For various piston speeds, the set of collapse solutions again divides into those cases with rising accretion rates and those with declining rates. The dividing line between these two sets of solutions occurs for $d_{p} \sim 6 \times 10^{14}$ $\mathrm{cm}$ for the piston velocities considered.

We examine the above results in more detail. The condition which determines which of the two classes of solutions a given collapse calculation will follow is not easily defined. First, the amount of work done by the piston during the push phase of the calculation will not alone determine the subsequent evolution of the collapse. Having taken the gas to be isothermal implicitly assumes that the cloud can instantaneously exchange energy with some external sources and sinks. As the piston pushes the gas outward, compressed regions of the gas will radiate away energy. Thus, the change in the energy of the cloud during the time that the piston is pushing does not depend solely on the amount of work done by the piston, but

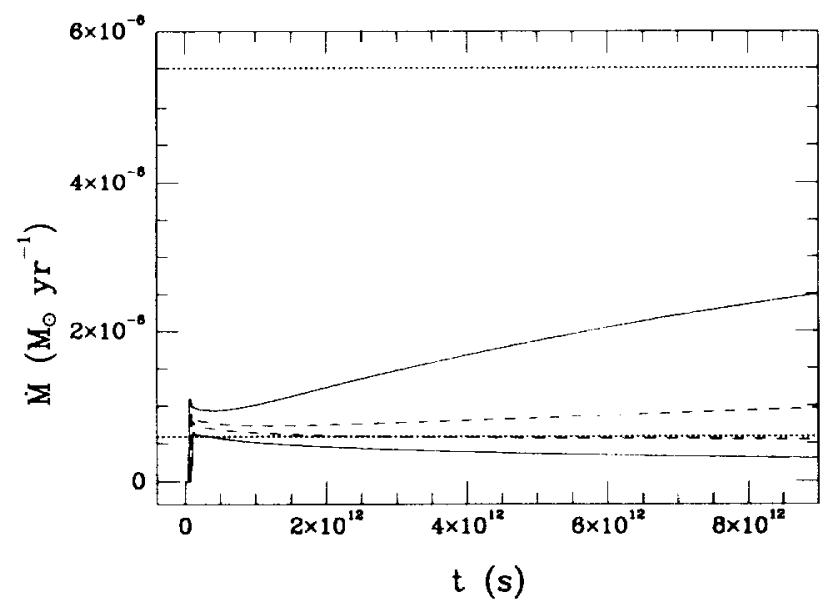

Fig. 5.-The central mass accretion rates for several cases are plotted as in Fig. 3. The solid lines give the case where $v_{p}=a$. The upper solid line assumes $d_{p}=4.7 \times 10^{14} \mathrm{~cm}$, and the lower one assumes $d_{p}=7.4 \times 10^{14} \mathrm{~cm}$. The dashed curves give the case where $v_{p}=1.2 a$. The upper curve assumes $d_{p}=5.9$ $\times 10^{14} \mathrm{~cm}$, and the lower curve assumes $6.3 \times 10^{14} \mathrm{~cm}$. The dotted lines give the accretion rates of the EWS (top line) and the shock solution (bottom line). also on its exact velocity history. Also, the collapse after the piston has been stopped is not energy conserving, again since the gas is assumed to be isothermal. Whatever changes in the energy of the cloud occurred during the time the piston was pushing are swamped by subsequent energy exchanges of the cloud with the external sources and sinks.

An alternative is to consider the dynamics of the shock. The shock is launched by the piston and grows in strength as the piston pushes outward. However, when the piston is stopped and the inner boundary condition is applied, a rarefaction wave is also sent propagating into the gas because the velocity of the gas just in front of the piston is positive when the piston is stopped. This can be seen in the topmost curve (corresponding to the earliest time shown) of Figure $2 b$. The shock is at a radius of $\sim 10^{16} \mathrm{~cm}$, and a rarefaction wave is seen at a radius of $\sim 6.3 \times 10^{15} \mathrm{~cm}$. A rarefaction wave travels at the local sound speed. Since the rarefaction wave generated by the piston propagates into a region of outflowing gas, the wave can actually catch up to the shock, even though the shock is traveling outward supersonically. This can be seen in both Figures $1 b$ and $2 b$. The rarefaction wave which is well separated from the shock at the earliest time plotted has caught up to the shock by the next time shown and is interacting with the shock and decreasing its strength. Once caught up with the shock, the rarefaction wave cannot proceed further upstream since the shock is propagating supersonically into a static medium. The subsequent evolution of the shock and the collapse solution in general then depends on the complicated nonlinear interactions of the rarefaction wave and the shock. The amplitudes of the shock and the rarefaction wave again depend in detail on the velocity history of the piston.

Although complex, there is hope that the late time behavior of the collapse can be understood. The evolution toward the EWS of collapse initiated by central perturbations without accompanying piston motions suggests that there must be some link between the late time, scale-free evolution of our collapse solutions with a similarity solution. Analogous considerations based on the Sedov-Taylor problem further emphasize this link. What then should be the properties of such a similarity solution? The numerical considerations indicate that if the actions of the piston are very finely tuned, a collapse which has properties between those of the two numerical classes can be obtained. Specifically, the solution would have a constant central accretion rate and an approximately Mach 1.26 constant amplitude shock propagating outward. We find such a solution in $\S 3$ and use it to understand the late time evolution of the numerical solutions.

\section{SIMILARITY SOLUTIONS}

\subsection{The Shock Solution}

The equations governing an isothermal gas sphere are the continuity equation,

$$
\frac{\partial \rho}{\partial t}+\frac{1}{r^{2}} \frac{\partial}{\partial r}\left(r^{2} \rho u\right)=0,
$$

and the Euler equation,

$$
\frac{\partial u}{\partial t}+u \frac{\partial u}{\partial r}=-\frac{a^{2}}{\rho} \frac{\partial \rho}{\partial r}-\frac{G M}{r^{2}},
$$

where $\rho$ is the gas density, $u$ is the gas velocity, $M$ is the mass enclosed within radius $r$, and $a$ is again the isothermal sound 
speed. Following Shu (1977), we define the similarity variable

$$
x=\frac{r}{a t},
$$

and we nondimensionalize the fluid variables,

$$
\begin{gathered}
\rho(r, t)=\frac{\alpha(x)}{4 \pi G t^{2}}, \\
M(r, t)=\frac{a^{3} t}{G} m(x), \\
u(r, t)=a v(x) .
\end{gathered}
$$

Equation (6) indicates that the mass is zero at the instant $t=0$. This corresponds to the moment of core formation. We will consider positive times after the formation of the core. Substituting equations (4)-(7) into equations (2) and (3), we get the following set of equations (same as eqs. [11] and [12] of Shu 1977),

$$
\begin{gathered}
{\left[(x-v)^{2}-1\right] \frac{d v}{d x}=\left[\alpha(x-v)-\frac{2}{x}\right](x-v),} \\
{\left[(x-v)^{2}-1\right] \frac{1}{\alpha} \frac{d \alpha}{d x}=\left[\alpha-\frac{2}{x}(x-v)\right](x-v),}
\end{gathered}
$$

for the nondimensionalized fluid variables. It can also be shown using the continuity equation for $M(r, t)$ rather than for the density that the nondimensionalized mass is given by

$$
m=x^{2} \alpha(x-v) \text {. }
$$

Shu (1977) found a large set of solutions to equations (8)(10), some of which were identified as viable collapse solutions. These were called "minus solutions without critical points" (MSWCPs) (see Fig. 2 of his paper and the light dotted lines of Figs. 6 and 7), of which the EWS represents a certain limit (although the EWS is not one of the MSWCPs). The MSWCPS correspond to solutions of equations (8)-(10), where the outer boundary condition requires that the gas be static at large $x$ and the inner boundary condition requires that the gas be in freefall,

$$
m \rightarrow m_{0}, \quad \alpha \rightarrow\left(m_{0} / 2 x^{3}\right)^{1 / 2}, \quad v \rightarrow-\left(2 m_{0} / x\right)^{1 / 2} \quad \text { as } x \rightarrow 0,
$$

where $m_{0}$ is the nondimensionalized mass of the accreting central protostar. These solutions are so dubbed because the entire flow from the outer static region to the inner free-falling regime occurs without passing through a critical point. Note that the coupled set of equations (8) and (9) have a locus of critical points given by

$$
x=1+v,
$$

which is shown as the dashed line of Figure 6. The entire set of MSWCPs does not intersect this line.

The MSWCPs correspond physically to the collapse of initially static isothermal spheres with $r^{-2}$ density profiles but which are too dense to be in equilibrium. The initial density can be represented by

$$
\rho(r, 0)=\frac{a^{2} A}{4 \pi G} r^{-2}
$$

where $A$ is a numerical constant satisfying the condition $A>2$.

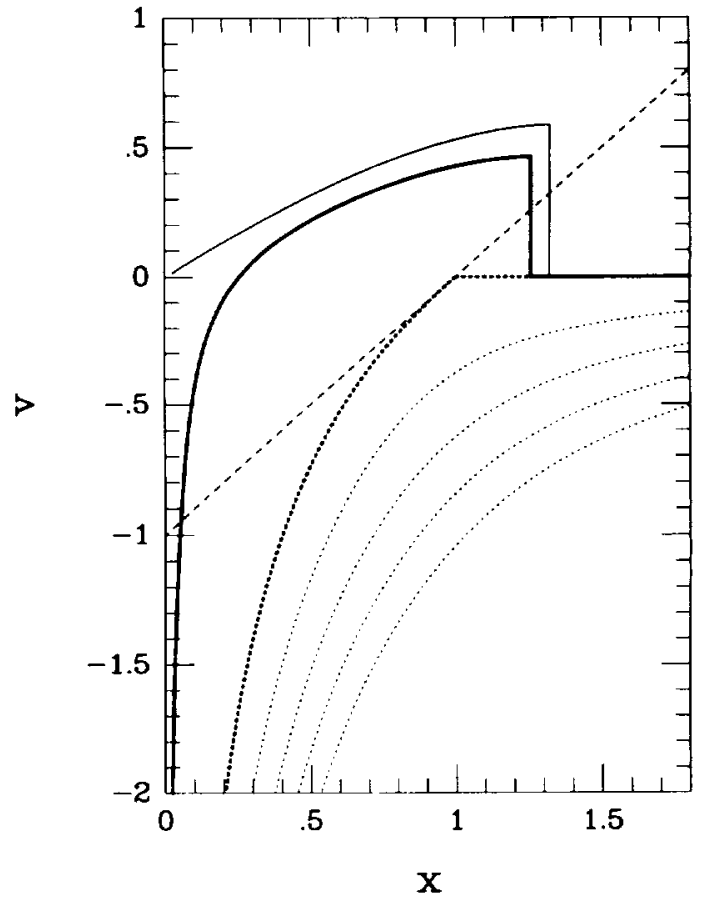

FIG. 6.-The nondimensionalized velocities for various similarity solutions are plotted vs. the similarity variable. The light dotted lines give the "minus solutions without critical points" (MSWCPs). From the lowest to the uppermost curve, these correspond to $A=2.8,2.6,2.4$, and 2.2 . The heavy dotted line gives the expansion wave collapse solution (EWS; $A=2$ ), and the heavy solid line gives the shock solution. The light solid line gives the expanding solution, and the dashed line gives the locus of critical points of eqs. (8) and (9) of the text.

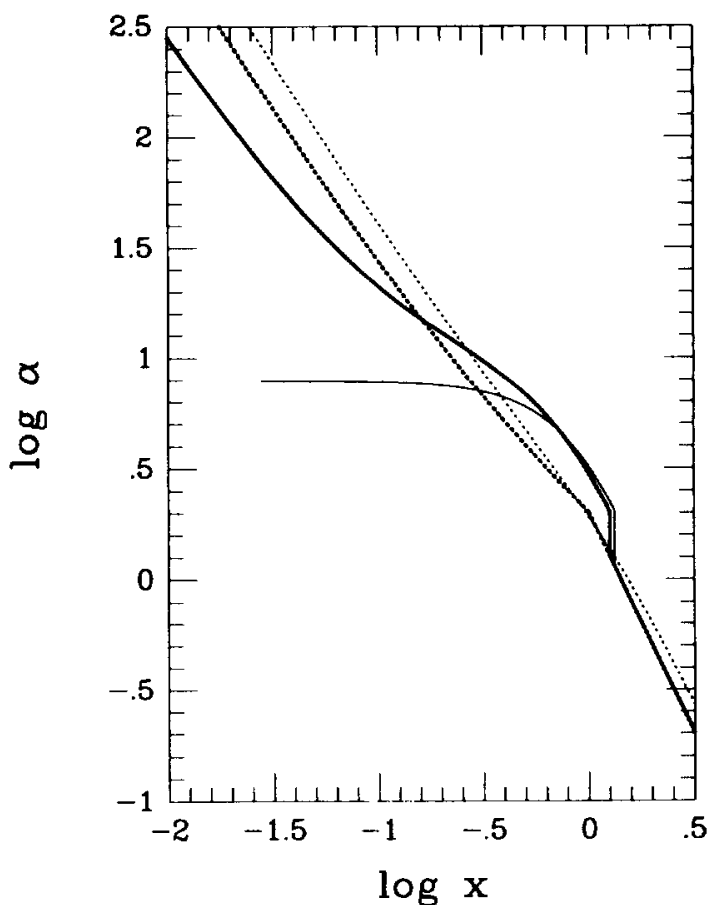

FIG 7-The nondimensionalized density profiles for several similarity solutions are plotted. The light dashed line gives the MSWCPs corresponding to $A=2.8$, and the heavy dotted line gives the expansion wave collapse solution (EWS; $A=2$ ). The heavy solid line gives the shock solution, and the light solid line gives the expanding solution. 
The limiting case of $A=2$ corresponds to the singular isothermal sphere, implying that the EWS is the limit of the MSWCP solutions as $A \rightarrow 2$. The values of $m_{0}$ are determined uniquely by the value of $A$. For the EWS, $m_{0}=0.975$, and it is larger for solutions with larger values of $A$.

The EWS obeys the same inner and outer boundary conditions as the MSWCPs; however, the flow passes through a critical point at $x=1$ (Fig. 6). This implies that the EWS is actually a member of a different set of solutions called "minus solutions" (Shu 1977). These are solutions which obey the inner boundary condition of equation (11) (with values of $m_{0} \leq 0.975$ ) but which do pass through a critical point with positive slope in the velocity profile. With the exception of the EWS, the minus solutions were originally discarded as inappropriate for collapse since the inner inflowing part of the solution could not be joined consistently with an outer solution which obeyed the static outer boundary condition. More specifically, starting with values given by the inner boundary condition (11), the fluid variables can be integrated outward to the location of the critical line (e.g., the negative velocity portion of the heavy solid line in Fig. 6 is a minus solution). In order that the flow then passes smoothly through the critical point with positive slope in the velocity profile, equations (8) and $(9)$ require that

$$
\begin{gathered}
-v=\left(1-x_{*}\right)-\frac{1}{x_{*}}\left(x-x_{*}\right)+\cdots, \\
\alpha=\frac{2}{x_{*}}-\frac{2}{x_{*}^{2}}\left(x-x_{*}\right)+\cdots,
\end{gathered}
$$

where $x_{*}$ is the position of the critical point (the value of $x_{*}$ is fixed by the value of $m_{0}$ or vice versa). The fluid variables can then be integrated outward to the point where the gas velocity is zero. Here the velocity profile of the inner solution can be smoothly joined to the static outer part. However, the density at this point from the inner integration does not correspond to the density appropriate to that radius in the singular isothermal sphere. The velocity and density therefore cannot be simultaneously matched to a static outer solution. The EWS is the only exception that allows a consistent matching.

Guided by the numerical considerations of $\$ 2$, the presence of a shock somewhere in the flow allows for 1 more degree of freedom (the location of the shock) which might allow the fluid variables of the inner part of a minus solution to be consistently joined via the shock jump conditions to a static outer solution. We first determine the appropriate shock jump conditions. Since we consider an isothermal shock, we need only consider mass and momentum conservation across the shock. If we assume that the shock is propagating into static gas, we have, in terms of dimensional fluid variables,

$$
u_{2}=\frac{u_{\mathrm{sh}}^{2}-a^{2}}{u_{\mathrm{sh}}}, \frac{\rho_{2}}{\rho_{1}}=\left(\frac{u_{\mathrm{sh}}}{a}\right)^{2},
$$

where $u_{2}$ and $\rho_{2}$ are the postshock gas velocity and density, respectively, $u_{s h}$ is the speed of the shock as it goes into the static gas, and $\rho_{1}$ is the preshock gas density. Assuming the shock travels at a constant speed and starts at the origin at $t=0$, the location of the shock expressed in terms of the similarity variable will be fixed. That is, $u_{\mathrm{sh}}=x_{\mathrm{sh}} a$, where $x_{\mathrm{sh}}$ is the fixed position of the shock in similarity space. The jump conditions in nondimensional form are then

$$
v_{2}=x_{\mathrm{sh}}-\frac{1}{x_{\mathrm{sh}}}, \quad \alpha_{2}=2 \text {, }
$$

where again a subscript of 2 denotes postshock values. In deriving equation (16), we have taken the preshock density to be that of the singular isothermal sphere.

The property that $\alpha_{2}=2$ at the shock suggests the following scheme for determining the desired shock solution. Pick a location on the line of critical points such that $x_{*}$ lies in the range $0<x_{*}<1$. The values of the fluid variables at $x_{*}$ are then fixed by equation (14). Using these as starting values for an integration to smaller $x$ yields the value of $m_{0}$ which corresponds to the assumed value of $x_{*}$. The fluid variables are then integrated outward from $x_{*}$ to the point where $\alpha_{2}=2$. Take this as the location of the shock $x_{s \mathrm{~s}}$. The jump condition for the density is then automatically satisfied at this point. However, the velocity jump condition may not be simultaneously satisfied. The initially assumed value of $x_{*}$ is adjusted and the above procedure repeated until both jump conditions are simultaneously satisfied.

We find that there is a unique value of $x_{*}$ (hence also of $m_{0}$ and $x_{\mathrm{sh}}$ ) such that the solution both passes smoothly through the critical point and satisfies the shock jump conditions, given that the outer solution is the singular isothermal sphere. This solution is shown as the heavy solid line in Figures 6 and 7, and a listing of values is given in Table 1 . We get $x_{*}=0.0544$, which implies that $x_{\mathrm{sh}}=1.26$ and that $m_{0}=0.105$. That is, the shock has a Mach number of 1.26 , and the mass accretion rate onto the central "protostar" is constant and is given by equation (1) with $C=0.105$.

This solution represents the dividing line between the numerical class I and class II solutions. The predicted constant accretion rate neatly separates those cases with increasing acc-

TABLE 1

SHOCK SOLUTION*

\begin{tabular}{cccc}
\hline \hline $\begin{array}{c}c \\
(1)\end{array}$ & $\begin{array}{c}\alpha \\
(2)\end{array}$ & $\begin{array}{c}v \\
(3)\end{array}$ & $\begin{array}{c}m \\
(4)\end{array}$ \\
\hline $0.00 \ldots \ldots$ & $\infty$ & $-\infty$ & 0.105 \\
$0.05 \ldots \ldots$ & 40.0 & -1.03 & 0.108 \\
$0.10 \ldots \ldots$ & 21.2 & -0.446 & 0.116 \\
$0.15 \ldots \ldots$ & 15.7 & -0.218 & 0.130 \\
$0.20 \ldots \ldots$ & 13.0 & -0.0921 & 0.152 \\
$0.25 \ldots \ldots$ & 11.3 & -0.00891 & 0.182 \\
$0.30 \ldots \ldots$ & 10.0 & 0.0532 & 0.222 \\
$0.35 \ldots \ldots$ & 9.02 & 0.103 & 0.272 \\
$0.40 \ldots \ldots$ & 8.19 & 0.146 & 0.333 \\
$0.45 \ldots \ldots$ & 7.47 & 0.183 & 0.403 \\
$0.50 \ldots \ldots$ & 6.83 & 0.217 & 0.484 \\
$0.55 \ldots \ldots$ & 6.26 & 0.247 & 0.574 \\
$0.60 \ldots \ldots$ & 5.75 & 0.275 & 0.673 \\
$0.65 \ldots \ldots$ & 5.29 & 0.300 & 0.781 \\
$0.70 \ldots \ldots$ & 4.86 & 0.324 & 0.896 \\
$0.75 \ldots \ldots$ & 4.48 & 0.345 & 1.02 \\
$0.80 \ldots \ldots$ & 4.13 & 0.365 & 1.15 \\
$0.85 \ldots \ldots$ & 3.80 & 0.383 & 1.28 \\
$0.90 \ldots \ldots$ & 3.51 & 0.400 & 1.42 \\
$0.95 \ldots \ldots$ & 3.24 & 0.414 & 1.57 \\
$1.00 \ldots \ldots$ & 2.99 & 0.427 & 1.71 \\
$1.05 \ldots \ldots$ & 2.77 & 0.439 & 1.87 \\
$1.10 \ldots \ldots$ & 2.56 & 0.448 & 2.02 \\
$1.15 \ldots \ldots$ & 2.37 & 0.455 & 2.18 \\
$1.20 \ldots \ldots$ & 2.19 & 0.461 & 2.33 \\
$1.25 \ldots \ldots$ & 2.03 & 0.463 & 2.49 \\
\hline
\end{tabular}

" Col. (1) gives the position in similarity space, col. (2) is the nondimensionalized density, col. (3) is the nondimensionalized velocity, and col. (4) is the nondimensionalized enclosed mass. 
retion rates from those with declining rates (see Figs. 3 and 5). In addition, since the analytic "shock" solution is self-similar, the distance of the shock to the origin is a fixed multiple of the distance of the stagnation point to the origin. This property separates the class I solutions which have a decreasing value of the ratio of these two distances (Fig. 1a) from the class II solutions which have an increasing ratio (Fig. 1b). The character of the shock in the analytic solution is also comparable to that of the class II solutions, moving out at about the same rate and having constant amplitude.

\subsection{The Energetics of the Shock Solution}

We consider in more detail some of the properties of the shock solution. An obvious issue is what drives the large region of outflowing gas. This is most easily addressed by looking at how the energy of the solution evolves with time. In principle, the static singular isothermal sphere has infinitely negative total energy. This is because the total energy is the sum of the gravitational energy $\left(E_{\mathrm{grav}}\right)$ and the thermal energy $\left(E_{\mathrm{th}}\right)$, which are each given within a radius $r$ by

$$
\begin{gathered}
E_{\mathrm{grav}}(r)=-\int_{0}^{r} \frac{G M(r)}{r} \rho(r) 4 \pi r^{2} d r=-\frac{4 a^{4}}{G} r, \\
E_{\mathrm{lh}}(r)=\int_{0}^{r} \frac{3}{2}\left[\frac{\rho(r) k T}{\mu m_{p}}\right] 4 \pi r^{2} d r=\frac{3 a^{4}}{G} r,
\end{gathered}
$$

where $\mu m_{p}$ is the mean mass per particle. Clearly, if $r$ extends to infinity, the total energy is infinitely negative. Note that equations (17) and (18) are consistent with the virial theorem, since the singular isothermal sphere truncated at any finite radius is in equilibrium only if an external pressure acts at the outer boundary. A boundary term, which is customarily discarded in the statement of the theorem, must be included in our case. Consider now the energy of the flowing part of the shock solution. Within a fixed value of the dimensionless distance $x$,

$$
\begin{gathered}
E_{\mathrm{grav}}(x, t)=-\frac{a^{5} t}{G} \int_{0}^{x} x \alpha(x) m(x) d x, \\
E_{\mathrm{th}}(x, t)=\frac{3}{2} \frac{a^{5} t}{G}\left[m(x)-m_{0}\right],
\end{gathered}
$$

where the fluid variables are converted to the forms of equations (5) and (6). The quantity $m_{0}$ in equation (20) represents the mass accreted onto the core and is subtracted from the total enclosed mass, since the accreted gas is assumed to have no thermal effect on the collapse. We additionally need to consider the total kinetic energy of the flowing gas,

$$
E_{\mathbf{k i n}}(x)=\frac{a^{5} t}{2 G} \int_{0}^{x} \alpha(x) u^{2}(x) x^{2} d x
$$

The integrals of equations (19) and (21) can be evaluated numerically within the shock radius $x_{\mathbf{s h}}$. The total energy is then

$$
\begin{aligned}
E_{\mathrm{tot}}\left(x_{\mathrm{sh}}, t\right) & =E_{\mathrm{grav}}+E_{\mathrm{th}}+E_{\mathrm{kin}} \\
& =(-3.53+3.62+0.17) \frac{a^{5} t}{G}=0.26 \frac{a^{5} t}{G},
\end{aligned}
$$

which is positive. This implies that as the flowing region expands outward in time, the energy of each region that gets engulfed by the expanding shock increases. This behavior can be contrasted with that of the collapsing region of the EWS.
Equations (19)-(21) imply that inside of the rarefaction wave $x=1$, the energy is

$$
\begin{aligned}
E_{\mathrm{tot}}(x=1, t) & =E_{\mathrm{grav}}+E_{\mathrm{th}}+E_{\mathrm{kin}} \\
& =(-2.98+1.54+0.69) \frac{a^{5} t}{G}=-0.75 \frac{a^{5} t}{G} .
\end{aligned}
$$

We consider the flow of energy in these solutions. The isothermal nature of the gas does not derive from the gas being in thermodynamic equilibrium with a large heat reservoir. In the realistic setting of modeling the collapse of a cloud core, isothermality arises out of the stable balance between heating due to sources such as cosmic rays and cooling due to radiation from dust. For example, in the cases where the gas is heated by compression, dust emission is assumed to be so efficient that the extra heat can be radiated away rapidly. In the opposite case where gas is cooled by expansion, the various sources of heating are assumed to be sufficiently rapid to heat the gas back to the stable equilibrium temperature. In this sense, the shock solution does not represent a violation of the second law of thermodynamics. That is, if we had considered a cloud which was isothermal with temperature $T$ because it was in thermal contact with a bath with the same temperature, the extra energy of the expanding region would have represented the conversion to mechanical energy of heat without any accompanying work done on the system. However, this is not the case with the shock solution. The system is not in thermodynamic equilibrium with any thermal bath or radiation field. The temperature is determined only by the balance of a heating and cooling rate due to nonthermal processes which do not have the same temperature as that assumed for the cloud. In the case of the EWS, the pure compression in the flowing region of the cloud implies that no extra energy is taken in from the heating sources leading to a purely negative total energy. The motions of gas in the shock solution are more complicated. Initially, static gas is first compressed by the passage of the shock. The gas radiates compressional heat, but because this gas now has outward motion, it also cools by expansion. At some point, the expansion causes the temperature of the gas to want to dip below the equilibrium temperature appropriate to the heating and cooling rates. The gas then takes in extra energy from the heating sources to remain isothermal. Finally, as the parcel of gas is decelerated and sent into infall, compressional energy is again radiated by the gas. Because there is a net energy taken in by the gas, the energy of the flowing region is positive and increasing.

In the realistic setting of a finite-sized cloud core, collapse described by the shock solution would eventually make the total energy of the core positive, hence making it unbound. This is acceptable behavior in light of the instability of the singular isothermal sphere to both collapse and expansion. The shock solution simply represents a mode of collapse which allows the accretion of some material onto a core but which at the same time interacts with an external energy source so as to make the flowing parts of the gas unbound. To emphasize this point, we demonstrate in $\S 4$ that the singular isothermal sphere can be easily sent into complete expansion if collapse is not initially forced on the cloud.

We note that the above energy considerations apply equally well to the shock found in the numerical solutions of $\S 2.2$. The important thing to consider in that case is that the shock persists long after the piston has been applied to the gas. The energetics of the postshock gas are only related to the actions 
of the piston in the sense that the piston puts the gas into a state where the subsequent evolution is driven by a shock and the requirement that the gas be isothermal. The work done by the piston is insignificant compared to the subsequent energy exchanges of the cloud in trying to remain isothermal.

We have assumed that the rate at which the cloud is heated in the expanding part of the flow is sufficiently rapid so that there will only be small departures from isothermality. A concern might be that the evolution of the gas in the shock solution may require a heating rate which is so high that the prescribed processes cannot supply the needed power. This is not likely the case since the shock in the above solution is weak; the postshock motions are subsonic, and the density contrast at the shock is only a factor $\sim 1.6$. We examine this quantitatively. During the early phases of collapse as described by the shock solution, the shock is in the high-density region near the core. This part of the collapse is not well modeled by any isothermal collapse solution since the gas is expected to be hotter due to trapped compressional heat. At somewhat later times, the shock will be in a more optically thin region where grain cooling will be more effective and where isothermal solutions may apply. Heating in this region can be due to absorption of accretion luminosity, cosmic-ray impacts, and grain heating. The latter two processes are expected to be most important in the outer, low-density regions of the cloud, whereas the effects of accretion luminosity are most important in the inner regions (although significant heating from accretion luminosity occurs even when the gas is optically thin; Hollenbach et al. 1995; Ceccarelli, Hollenbach, \& Tielens 1995). In Figure 8, we show estimates of heating rates due to the aforementioned processes as well as the rate of adiabatic cooling due to the expansion of gas immediately behind the shock. (We do not include other forms of cooling since these are ineffective at temperatures of less than about $10 \mathrm{~K}$.) The cooling rate is given by

$$
\Lambda_{\mathrm{ad}}(r)=\frac{5}{2} \frac{k T u}{\mu m_{p}}\left(\frac{d \rho}{d r}\right)=-5 \frac{a^{2} u \rho}{r},
$$

where all fluid variables are dimensional. We have assumed

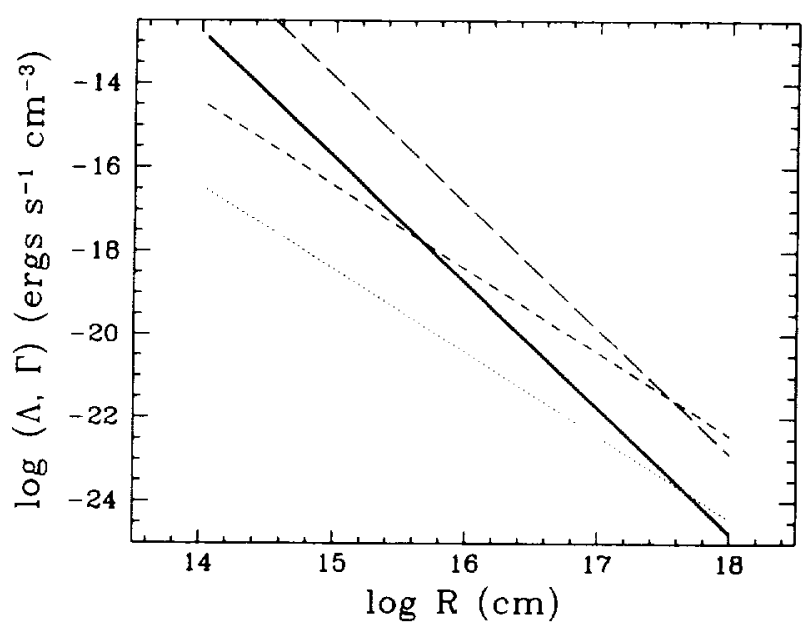

FIG. 8.- The magnitude of the rate of adiabatic cooling of gas expanding immediately behind the shock is given by the heavy solid line as a function of the position of the shock. The heating rates per unit volume of the same gas due to cosmic rays (dotted line) and dust heating (short-dashed line) are also plotted as a function of the location of the shock. The long-dashed line indicates the heating rate due to accretion luminosity. that the density distribution obeys $\rho \propto r^{-2}$ in obtaining the rightmost equality. We estimate the heating rates using

$$
\Gamma_{\mathrm{cr}} \sim 4 \times 10^{-28}\left(\frac{\rho}{\mu m_{p}}\right) \mathrm{ergs} \mathrm{s}^{-1} \mathrm{~cm}^{-3}
$$

for cosmic rays (Black 1987) and

$$
\Gamma_{\text {dust }} \sim 4 \times 10^{-26}\left(\frac{\rho}{\mu m_{p}}\right) \text { ergs s}^{-1} \mathrm{~cm}^{-3}
$$

for photoelectric heating from dust (Black 1987). Heating from accretion luminosity is estimated by taking the luminosity to be

$$
L_{*}=\frac{G M_{*} \dot{M}}{R_{*}}, \quad R_{*} \sim 3 \times 10^{11}\left(\frac{\dot{M}}{10^{-5} M_{\odot} \mathrm{yr}^{-1}}\right)^{1 / 3} \mathrm{~cm},
$$

where the latter relation for $R_{*}$ is a fit to detailed calculations (Adams \& Shu 1985; Stahler, Shu, \& Taam 1980; Stahler 1983) and $\dot{M}$ is given by eq. (1) with $C=0.105$, the value appropriate to the shock solution. The mass of the star is just $M_{*}=\dot{M} t$, where $t$ is the time under consideration. The heating due to accretion luminosity for gas at radius $r$ is then computed:

$$
\Gamma_{\mathrm{acc}}=\kappa \rho\left(\frac{L_{*}}{4 \pi r^{2}}\right)
$$

where $\kappa$ is the absorption opacity of the gas. The dust photosphere which reprocesses the radiation from the accretion shock should have a temperature $\sim 500 \mathrm{~K}$ (Adams \& Shu 1985) which corresponds to a peak wavelength of $\sim 6 \mu \mathrm{m}$. The opacity here is $\kappa \sim 2.5 \mathrm{~cm}^{2} \mathrm{~g}^{-1}$. Ideally, $\kappa$ should be averaged over the emergent spectrum; however, we simply take the above value for our estimates. Equation (28) also represents an approximation since the emission from the dust photosphere will be further reprocessed by the material between the photosphere and the radius under consideration, $r$. If $r$ is reasonably small, then this should not be too bad an approximation since most of the energy fed in at the dust photosphere will eventually make it out to radius $r$.

The abscissa in Figure 8 is the location of the shock. The heating and cooling rates are computed for the gas immediately behind the shock because this is the region experiencing the greatest expansion. In the inner region $\left(r \sim 10^{15}-10^{16}\right.$. $\mathrm{cm}$ ) where both cosmic-ray and dust heating are minimized by the high optical depth, accretion luminosity provides an ample supply of energy to overcome the cooling due to expansion. In the outer region $\left(r \geq 10^{16.5} \mathrm{~cm}\right)$ where the amount of accretion luminosity absorbed by the gas is minimal, dust and cosmicray heating adequately replace the energy density lost via expansion.

\subsection{Radial Stability of the Shock Solution}

We examine the stability of the shock solution numerically. Although less satisfactory than linear stability analysis, essential features of the stability of the solution can be discerned in this manner. For example, when numerical calculations which begin with core collapse are continued beyond the point where the EWS is recovered, the subsequent evolution of the gas does not deviate substantially from the EWS (e.g., Boss \& Black 1982). This suggests that the EWS is stable to radial perturbations, a result also determined analytically (e.g., Silk \& Suto 
1988; Ori \& Piran 1988). We have also confirmed this result. We start a numerical calculation by assuming that the initial density and velocity profiles are those of the EWS scaled to some early time. The cloud is then evolved for about a dynamical time with the inner boundary condition that there be free fall onto a sink cell (the same boundary condition as adopted in $\$ 2$ ). We find no substantial deviation from the EWS.

The stability of the shock solution can be similarly tested. We set the initial density and velocity profiles of a numerical calculation to be that of the shock solution at some early time. We evolve the cloud with the same free-fall inner boundary condition as used previously. The subsequent collapse rapidly departs from that of the shock solution and suggests that it is unstable. We further test the behavior of the numerical collapse. Since we know from the analytic solution what the gas velocity should be at every location and time, we find that the infall speed of the shock solution at the radius where the inner boundary condition was imposed is somewhat smaller than the corresponding free-fall speed based on the enclosed mass. This is because pressure effects, though small, still contribute to the dynamics of the flow. In trying to eliminate all possible perturbations on the flow, we ran another numerical collapse calculation imposing the new inner boundary condition that the velocity and density be exactly as specified by the analytic solution at the given time and location. Even under these conditions, numerical noise is sufficient to drive the subsequent evolution of the gas away from that specified by the shock solution.

The difference in the stability properties of the EWS and the shock solution are not surprising. Within the rarefaction wave, the EWS possesses a large region of supersonic infall and steep velocity gradients which can serve to shear out perturbations. This stabilizes the flow (Shu 1977). The shock solution, on the other hand, has a large region of subsonic outflow which is accompanied by rather limited velocity gradients. Furthermore, the constant amplitude shock is maintained by a precarious interplay between a pure outgoing shock and a pure outgoing rarefaction wave. Perturbations to this arrangement in the form of sound waves can probably do considerable damage. A linear stability analysis of the shock solution will be presented elsewhere.

We studied the ways in which the numerical collapse departs from the shock solution by perturbing the flow away from the analytic solution in various ways. In the first instance, we again start the calculation by taking an early-time version of the shock solution as giving the initial density and velocity profiles for the gas. We also impose the free-fall inner boundary condition. However, we artificially increase the initial mass of the sink cell by $5 \%$. This has the effect of gravitationally perturbing the entire cloud. The collapse then proceeds according to the properties of the class I numerical solutions of $\$ 2.2$. More specifically, the central mass accretion rate increases rapidly from the value specified by the shock solution and approaches in time the value corresponding to the EWS solution. The amplitude of the shock decreases with time, and the ratio of the distances from the shock to the origin and the stagnation point to the origin decreases with time (i.e., the region of outflowing gas in similarity space gets smaller). Note that the same perturbation imposed on a numerical realization of the EWS does not result in departures from the analytic solution. In the second case, we run a collapse calculation in the same manner as above, but we instead artificially decrease the initial mass of the sink cell. We first decrease the mass of the sink cell so that the free-fall velocity at the radius where the inner boundary condition is applied is equal to the velocity as specified by the shock solution at that radius. The mass is then decreased a further $5 \%$ to run the calculation. The subsequent collapse evolves according to the class II solutions of $\S 2.2$. The central mass accretion rate decreases from the level specified by the analytic shock solution, and the shock persists in time with roughly constant amplitude.

The modes of instability described above provide an explanation for the existence of the two classes of collapse solutions found in the piston experiments of $\S 2.2$. After the collapse is launched by the piston and imposition of the inner boundary condition, the shock propagates out to a distance where the initial scale set by the piston motion becomes much smaller than the scale of the region over which the gas is flowing. The collapse attempts to go toward the shock solution; however, because it is unstable, perturbations imposed by the early piston motion nudge the collapse toward one of the two modes of instability growth. If the piston does not go out too far and does not impose initial conditions too far removed from simply having an initial pressure imbalance in the core, the solution follows the class I type of collapse and evolves toward the nearest stable solution, the EWS. On the other hand, with a sufficiently large push, the piston launches the collapse into the class II type of behavior, with the solution evolving toward lower accretion rates.

The piston experiments show that, given a reasonable amount of initial push by the piston, accretion would continue, although at low rates, for the duration of the collapse simulations. It is, however, interesting to consider the limiting case if the calculation were to be allowed to run indefinitely. Accretion will likely have stopped, and we can ask if there is some solution which represents pure outflow. That is, are there conditions under which the singular isothermal sphere could be sent into complete expansion? This is considered in the next section.

\section{OUTFLOW SOLUTION}

We initiated the numerical calculations of $\S 2.2$ by pushing a piston and then imposing a free-fall boundary condition at the end of the push. The boundary condition models a large pressure imbalance in the center of the cloud which essentially forces the inner region into collapse. In this section, we consider the case where a push on the gas by the piston is followed by a no-flow boundary condition, i.e., imposing the condition that the gas velocity be zero at the inner boundary.

The results of one of the numerical calculations are shown in Figures 9 and 10. The piston again forms a shock which propagates in time to large radius with constant amplitude. In contrast to the results of $\S 2.2$, the velocity profile is one of pure outflow, and the shock has a higher Mach number of roughly 1.3. The density is characterized by a postshock region which flattens out toward small radius, in contrast to the steeply peaked density of the initial static isothermal sphere and to the density of the shock collapse solution. Identical results to those displayed here are obtained assuming any of a series of piston speeds and distances $\left(v_{p}\right.$ and $\left.d_{p}\right)$. In particular, the case shown in the figures assumes $v_{p}=0.5 a$ and $d_{p}=2 \times 10^{14} \mathrm{~cm}$. Recall that the case from $\S 2.2$ with the same piston parameters, but where the free-fall inner boundary condition was imposed, led to an increasing mass accretion rate and a diminishing shock (Figs. $1 a, 2 a$, and 3). In the present calculation, the shock does not weaken with time. Other cases where $d_{p}$ is even smaller 
(representing very small perturbations to the flow) also lead to constant amplitude shocks. Therefore, for the pure expansion case, there is no bifurcation into two classes of solutions. All piston parameters considered lead to the same solution as shown in Figures 9 and 10. Furthermore, only very modest initial perturbations are required to cause the singular isothermal sphere to expand.

The character of the numerical results again suggests that we seek a similarity solution to describe the late time evolution of the expanding solution. We use the formulation of $\$ 3.1$. The shock is again expanding into a static singular isothermal sphere. The shock must then obey the jump conditions of equation (16). Our procedure is first to assume a location for the shock, $x_{s h}$. Starting with values of the density and velocity given by equation (16), we integrate the fluid equations (8) and (9) numerically to small radius. The resulting gas velocity will not vanish in general as $x \rightarrow 0$. However, by adjusting $x_{\mathrm{sh}}$, this condition can be satisfied. We find that such a solution is unique, as was the case with the shock collapse solution of $\S 3.1$. The velocity and density profiles are given in Figures 6 and 7 , respectively, by the light solid line. The shock propagates outward at a constant speed of $1.34 a$.

To better compare the analytic result to the numerical solutions, we scale the similarity solution to the time of the last curve in Figures 9 and 10. The similarity solution is given by the dots. The numerical results agree exactly with the similarity solution, except of course for the shock which occupies several zones in the numerical simulation. This indicates both that the similarity solution gives a good description of the flow and that the solution is probably stable.

It is easy to show from equations (8) and (9) that

$$
\frac{d \alpha}{d x} \rightarrow 0 \text { as } x \rightarrow 0 \text {. }
$$

The passage of the shock through the singular isothermal sphere rearranges the gas into a flat distribution. The centrally peaked nature of the initial cloud is completely disrupted as gas is sent outward. Figure 7 shows that $\sim 30 \%$ of the expanding region is included in such a "core." Furthermore, equation

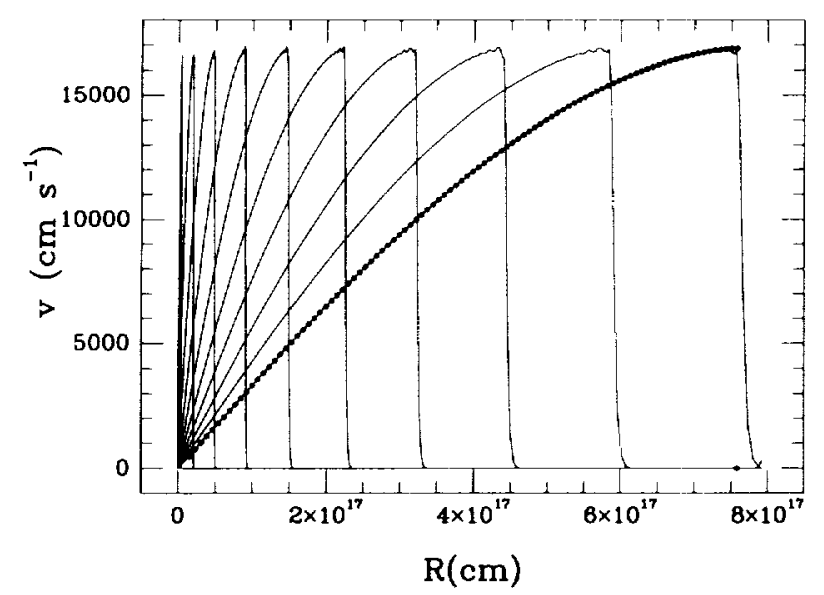

Fig. 9.-The gas velocities in the pure expansion case where $v_{p}=0.5 a$ and $d_{p}=2 \times 10^{14} \mathrm{~cm}$ are plotted for several times. The leftmost curve corresponds to the earliest time of $0.1 \times 10^{12} \mathrm{~s}$. Going toward the right, the corresponding times are $0.53,1.27,2.37 .3 .89,5.89,8.44,11.6,15.4$, and 19.9 in units of $10^{12} \mathrm{~s}$. The dots give the analytic solution of Fig. 6 scaled to the time of the last numerical calculation shown.

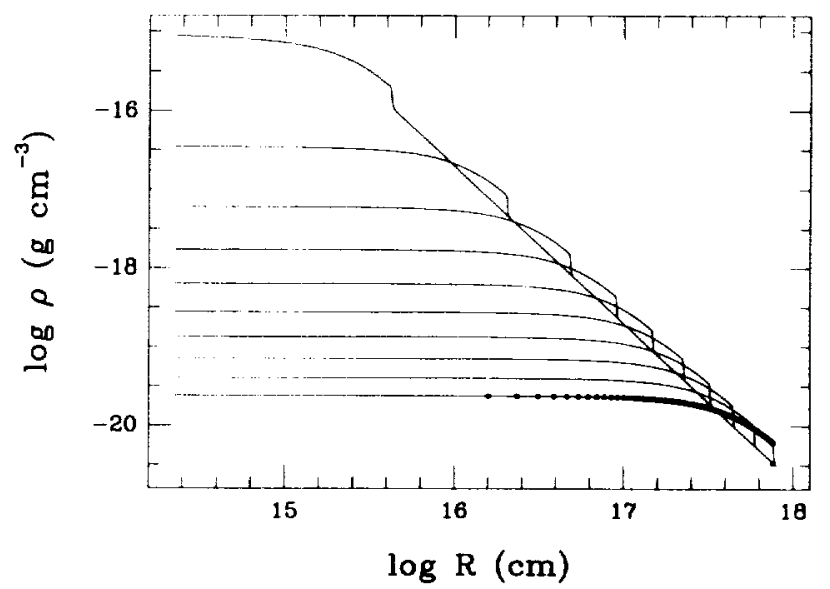

Fig. 10.-The gas density in the case of pure expansion where $v_{p}=0.5 a$ and $d_{p}=2 \times 10^{14} \mathrm{~cm}$ is plotted for the same times as shown in Fig. 9 . The dots indicate the analytic solution of Fig. 7 scaled to the time of the last numerical calculation shown

(5) indicates that the central density decreases as the square of time. After a time of $10^{5} \mathrm{yr}$, the central density is $\sim 2 \times 10^{5}$ $\mathrm{cm}^{-3}$, which is comparable to the density of a molecular cloud core (e.g., Goldsmith 1987). The expanding solution then not only demonstrates that the singular isothermal sphere can be easily sent into pure expansion, but that a very modest central perturbation and subsequent subsonic motions of the gas can lead to a uniform density distribution throughout the region originally occupied by the singular isothermal sphere.

We again emphasize that the shock in the solution of this section is not powered by the piston, which stops after a very short time. The piston simply puts the gas into a state where the subsequent evolution is governed by the dynamics of the shock and the assumption that the gas is isothermal.

\section{DISCUSSION AND CONCLUSIONS}

We have considered the collapse of the singular isothermal sphere in the case where infall is initiated by some arbitrary process which, in addition to causing a central pressure imbalance, is accompanied by some input of heat or energy into the cloud. The rationale for this is to understand the possible effects on the collapse of either preheating by accretion luminosity or some other means of early energy input into the cloud, such as stellar winds. In order to be rather general and to understand the relevant physics, we modeled this process numerically with a one-dimensional hydrodynamical code where we initiated collapse with a central piston which first pushes at a constant speed out to some distance which is small relative to the size of the cloud. The piston is then stopped and a free-fall boundary condition is applied. This inner boundary condition acts to initiate collapse in the central regions. We assume isothermal conditions throughout.

Numerical collapse solutions in the piston experiments fall into two classes, depending upon how far the piston is allowed to push. Both classes are characterized by the presence of an initial shock created by the action of the piston, a region of outflowing gas just inside of the shock, and inflowing gas in the central regions. If the piston is pushed to less than a certain distance, the subsequent rate of mass accretion onto the core increases with time and approaches the constant value corresponding to the EWS. The shock decays with time and becomes a sonic pulse. The amount of outflowing gas also 
decreases with time. These were dubbed class I solutions and correspond to cases where, because the initial central perturbation was not very different from the conditions giving rise to the EWS, the subsequent collapse tended toward the EWS. In the cases where the piston was allowed to push further out, the accretion rate instead decreased with time. The initial shock persisted with roughly constant amplitude, and the region of outflowing gas grew with time. These were dubbed class II solutions. These two classes of solutions existed for any of the assumed piston speeds which included both subsonic and supersonic cases.

The interpretation of these results is given in terms of the instability of a similarity solution (the shock solution) which serves to divide the two classes of numerically determined solutions. The shock solution is characterized by a constant central mass accretion rate whose value exactly divides the class I accretion rates from the class II accretion rates (Figs. 3 and 5). The shock solution also has a constant amplitude shock and a constant ratio of the distances from the shock to the origin and the stagnation point to the origin. We examine the stability properties of the shock solution by starting a numerical collapse calculation with gas densities and velocities given by the shock solution scaled to some early time. The subsequent evolution of the cloud was found to always deviate from the analytic solution. (This contrasts with the behavior of the EWS.) This indicates that the solution is most likely unstable to radial perturbations. The manner of the deviation was such that a small perturbation of the flow toward increasing accretion rates (artificially increasing the mass of the core), resulted in a solution with the exact properties of the class I solutions. A perturbation in the other direction (artificially decreasing the mass of the core) resulted in evolution akin to that of the class II solutions. The interpretation of the numerical solutions is that when the shock has reached a distance far removed from the scales set by the actions of the piston, the solution tries to go to the shock solution. However, because it is unstable, initial perturbations due to the piston cause the solution to evolve away from the shock solution in either the manner of the class I or class II solutions.

The shock solution and the class II numerical solutions eventually lead to the unbinding of any finite region of the singular isothermal sphere. The energy for this does not come from the actions of any piston. Rather, in the case of the numerical solutions, the piston changes the state of the cloud so that it absorbs extra energy from whatever agent is helping to keep the cloud isothermal. In the case of an actual protostellar cloud core, this would presumably include dust and cosmicray heating. Furthermore, this mode of collapse does not represent a violation of the instability properties of the singular isothermal sphere since it is unstable both to collapse and to expansion. The limit of the class II solutions is in fact represented by the case of zero mass accretion and pure outflow. We also obtained such a solution both numerically and analytically. The numerical realization is obtained by again pushing a piston out some distance into an initially static singular isothermal cloud and imposing the inner boundary condition that the velocity be zero at small radius. This expanding solution is characterized by an outgoing constant amplitude shock and a flat central density profile. In approximately a dynamical time, the central density falls to values comparable to those of interstellar cloud cores. We find that even when the piston is pushed a very small distance, a purely expanding solution is always obtained. The numerical solution then evolves rapidly toward a similarity solution. Because the late time evolution of the numerical solutions does not deviate from the similarity solution, it is likely that the expanding solution is stable.

We consider the implications of the above results for star formation. First, the deposition of energy or pressure into the central regions of a collapsing protostellar cloud must occur to some extent. For example, if we adopt the standard paradigm of low-mass star formation (Shu et al. 1993), as infall is initiated in the center of a singular isothermal sphere, a core develops rapidly onto which material is accreted. The preheating effect of the accretion luminosity will modify the flow in the region of gas that is not yet in supersonic infall on a timescale very short compared to the dynamical time. This has the effect of giving the gas an outward push. Note that the preheating effect does not have to be so extensive as to include the more optically thin regions of the cloud. If preheating is sufficient to create a weak shock in the inner optically thick region, our considerations show that the shock can propagate to large radius simply because the gas is being kept relatively isothermal in the thin region. It is also known that protostellar winds occur very early on in the formation of the protostar (e.g., see Bally \& Lane 1991). Although these winds are thought to be initially highly collimated, they nevertheless can impart significant outward momentum to some of the gas. We have modeled all of these processes by a piston pushing in the center of a singular isothermal sphere. Although heuristic, this approach does give an indication of the nature of the subsequent collapse. Specifically, there is the possibility of accretion rates that are lower than the canonical value given by the EWS. Depending upon the degree of the initial perturbation, accretion rates could be a factor of 10 lower than the EWS value and evolve toward lower values, or they could be initially rather low and rise up to the EWS value. The present work does not predict the exact rate since the extent of the initial perturbation is not known.

The prediction of reduced accretion rates may be related to the "luminosity problem" of protostars. The observed luminosity function of embedded sources in several star-forming regions implies that the central accretion rates are generally $\sim 10$ times lower than predicted by the standard paradigm (e.g., Kenyon et al. 1990; Kenyon et al. 1994; Greene et al. 1994). This discrepancy may be due to rotational effects that are not considered in the spherically symmetric approximation of the EWS (Kenyon et al. 1994). For example, most collapsing material could fall onto a disk and then be accreted onto the star on a timescale set by disk physics. The accretion from a disk could be, for the most part, smaller than that of spherically symmetric accretion. Since studies show that the lifetime of the accretion phase is $\sim 10^{5} \mathrm{yr}$, accretion from the disk must occur in a series of bursts separated by a phase of low accretion in order that the average accretion rate be high enough to form a solar-mass star in the given time. Our study indicates that independent of rotational considerations, which must be important to some degree, spherically symmetric collapse itself gives rise to time variable accretion rates which could be rather low, especially at early times. The objection is then raised that in $\sim 10^{5} \mathrm{yr}$ a consistently low accretion rate leads to a star that is well below a solar mass. There are several possible ways out of this. A time-variable accretion rate, such as those of the class I solutions, would imply that the low-luminosity sources are simply young and are experiencing a period of rather low accretion. At later times, the accretion rate increases so that a 
solar-mass star can still be formed in approximately the allowed time. On the other hand, if indeed the accretion rate remains consistently low, in $\sim 10^{5}$ yr the shock of our solutions would have traveled to the outer regions of the cloud core. The nature of the collapse after this depends in part on what happens to the shock on reaching the outer boundary, although the presence of significant magnetic support for the cloud in these regions will lessen the effects of the shock. Since this is unknown, what happens to the accretion rate after the first $\sim 10^{5}$ yr is unknown also.

The application of our study to protostellar winds is more heuristic. Early stellar winds are thought to be highly collimated and rather supersonic. Our solutions, however, are spherically symmetric, and the shock is rather weak. Therefore, our considerations will not provide an exact description of the effects of the wind on the collapsing cloud. However, we showed that the singular isothermal sphere is, qualitatively speaking, rather loosely bound; small initial perturbations toward expansion can easily lead to the general expansion of the cloud. This indicates that the termination of accretion can be easily effected by a stellar wind. Conversely, the star formation scenario might be that protostellar collapse first proceeds according to one of our solutions. The passage of the shock unbinds much of the material in the outer regions of the core. The onset of an observable protostellar wind may then occur as a result of the cloud no longer presenting much of a barrier against which the wind must fight to get out of the core.

The results of $\S 4$ also offer the intriguing possibility that if some process exists which applies a small push in the central regions of a centrally peaked cloud core without an accompanying pressure imbalance, the core can be easily dispersed in a dynamical time. It is not clear, however, whether such a process exists in nature.

In considering the relevance of the current study to star formation, we must be mindful of the limitations of our assumptions and methods. For example, the assumption that protostellar collapse occurs in an isothermal fashion has previously only been made in connection with a purely collapsing cloud. Under this latter condition, compressional heat generated during collapse is efficiently radiated by various mechanisms, including dust emission. However, our solutions include an expanding outer envelope which is cooled by expansion. We should therefore check that sufficient heating exists from various processes (cosmic rays, dust heating, and heating from accretion luminosity) to keep the gas isothermal. We have demonstrated that this is indeed the case by estimating the various heating rates and comparing them to the rate of adiabatic cooling of expanding gas (see $\$ 3.2$ ).

Another assumption of the current work is that the cloud into which the shock propagates is exactly stationary. If the currently held paradigm for low-mass star formation does indeed provide a framework for collapse (Shu et al. 1993), there could be a subsonic inward drift of the gas at large radius as the magnetic field diffuses out of the collapsing cloud. What is the behavior of the shock solution under these conditions or in the more extreme case where the inward drift is supersonic? It is obvious, since the shock in our solution has a Mach number of only 1.26 , that any inflow upstream of the shock of greater than the indicated speed will sweep the shock into the core. The solutions investigated in this paper would not apply to these flows. In the case where the inflow ahead of the shock is subsonic, the effects of our solution are somewhat reduced. For example, we have considered the collapse of isothermal spheres which have a density that is everywhere greater by a uniform factor than that of the singular isothermal sphere. Because these spheres are too dense to be in equilibrium, infall is initiated at all radii at the beginning of collapse. The case where infall is not accompanied by any central energy input is described by the MSWCP solutions discussed in $\S 3.1$. When a central piston is applied, we find that resulting solutions are similar to those in which a shock propagates into static gas; however, there is a greater tendency to follow the class I behavior (see $\S 2.2$ ). Specifically, the accretion rate, which always begins at rather low values $\left(\dot{M} \sim\right.$ few $\left.\times 10^{-7} M_{\odot} \mathrm{yr}^{-1}\right)$ shows a greater tendency to evolve toward the higher value associated with the corresponding MSWCP solution. The low accretion rates characterizing the early parts of the collapse of some of the class I solutions last for shorter times. Similarly, the magnitude of the shock tends to decrease more rapidly as does the size of the region of outflowing gas. However, even when gas ahead of the shock is infalling subsonically, significant reductions in the central accretion rate relative to the values associated with no energetic input are possible.

Current observational constraints on the nature of infall are inadequate for differentiating between our solutions and other models. Although molecular line width studies have ruled out outflow in various cloud cores (e.g., Zhou 1992; Evans et al. 1992), the nature of the infall has not been especially well constrained. Although collapse according to the Larson-Penston model (Larson 1969; Penston 1969a) is likely ruled out, the nature of the infall predicted by the shock solution is not very different from that of the EWS, in that free-fall will prevail in the central regions. Additionally, the outflows in our solutions are rather subsonic, so they are not detectable using line profiles. The constraints on the density profile are similarly ambiguous enough to allow a variety of models. Studies of spectral energy distributions indicate the density can be consistent with $\rho \sim 1 / r^{(1.5-2.0)}$ profiles (Butner et al. 1990; Butner et al. 1991). Because the EWS and our solutions both derive from collapsing a singular isothermal sphere, comparable density profiles exist throughout much of the cloud.

We thank P. Cassen for numerous useful discussions, encouragement, and for suggesting that we consider a similarity solution for understanding the numerical results. We also thank S. Stahler, F. Shu, P. Bodenheimer, D. Hollenbach, C. McKee, C. Ceccarelli, and T.-Y. B. Yang for many comments and discussions. Part of this work has been conducted under the auspices of the NASA astrophysics theory program, which supports the Center for Star Formation Studies at NASA/Ames Research Center, University of California, Berkeley, and University of California, Santa Cruz. J. C. T. was supported by an NRC associateship.

\section{REFERENCES}

Adams, F. C., \& Shu, F. H. 1985, ApJ, 296,655

Bally, J., \& Lane, A. P. 1991, in The Physics of Star Formation and Early Stellar Evolution, ed. C. J. Lada \& N. D. Kylafis (Dordrecht: Kluwer), 471

Black, J. H. 1987, in Interstellar Processes, ed. D. J. Hollenbach \& H. A Thronson (Dordrecht: Reidel), 731
Bodenheimer, P., \& Sweigart, A. 1968, ApJ, 152, 515

Bodenheimer, P., Yorke, H. W., Róźyczka, M., \& Tohline, J. E. 1990, ApJ, 355 651

Bonner, W. B. 1956, MNRAS, 116, 351

Boss. A. P., \& Black, D. C. 1982, ApJ, 258, 270 
Butner, H. M., Evans, N. J., Harvey, P. M., Mundy, L. G., Natta, A., \& Randich, M.S. 1990, ApJ, 364, 164

Butner, H. M.. Evans, N. J., Lester, D. F., Levreault, R. M., \& Strom, S. E. 1991, ApJ, 376,636

Ceccarelli, C., Hollenbach, D., \& Tielens, A. G. G. M. 1995, preprint

Ebert, R. 1955, Z. Astrophys., 37, 217

Evans, N. J., Zhou, S., Kömpe, C., \& Walmsley, C. M. 1992, in Planetary Systems: Formation, Evolution, and Detection, ed. B. F. Burke, J. H. Rahe, \& E. E. Roettger (Dordrecht: Kluwer), 139

Foster, P. N., \& Chevalier, R. A. 1993, ApJ, 416, 303

Galli, D., \& Shu, F. H. 1993a, ApJ, 417, 220 $1993 \mathrm{~b}, \mathrm{ApJ}, 417,243$

Gaustad, J. E. 1963, ApJ, 138, 1050

Goldsmith, P. F. 1987, in Interstellar Processes, ed. D. J. Hollenbach \& H. A. Thronson (Dordrecht: Reidel), 51

Greene, T. P., Wilking, B. A., André, P. A., Young, E. T., \& Lada, C. J. 1994, ApJ, 434, 614

Hollenbach D Ceccarelli, C Neufeld, D. \& Tielens, A. G. G. M. 1995, in Proc. Airborne Astronomy Symp. on the Galactic Ecosystem: From Gas to Stars to Dust, ed. M. Haas, J. Davidson, \& E. Erickson (San Francisco: ASP), in press

Hunter, C. $1977, \mathrm{ApJ}, 218,834$

Kenyon, S. J., Gomez, M., Marzke, R. O., \& Hartmann, L. W. 1994, AJ, in press
Kenyon, S. J., Hartmann, L. W., Strom, K. M., \& Strom, S. E. 1990, AJ, 99, 869 Larson, R. 1969, MNRAS, 145, 271

Morfill, G. E., Tscharnuter, W., \& Völk, H. J. 1985, in Protostars and Planets II, ed. D. C. Black \& M. S. Mathews (Tucson: Univ. Arizona Press), 493

Ori, A., \& Piran, T. 1988, MNRAS, 234, 821

Penston, M. V. 1969a, MNRAS, 144, 425

1969b, MNRAS, 145, 457

Sedoy, L. I. 1959, Similarity and Dimensional Methods in Mechanics (New York: Academic)

Shu, F. H. 1977, ApJ, 214, 488

Shu, F. H., Adams, F. C., \& Lizano, S. 1987, ARA\&A, 25, 23

Shu, F. H., Najita, J., Galli, D., Ostriker, E., \& Lizano, S. 1993, in Protostars and Planets III, ed. E. H. Levy \& J. I. Lunine (Tucson: Univ. Arizona Press) 3

Silk, J., \& Suto, Y. 1988, ApJ, 335, 295

Stahler, S. W. 1983, ApJ, 274, 822

Stahler, S. W., Shu, F. H., \& Taam, R. E. 1980, ApJ, 241, 637

Terebey, S., Shu, F. H., \& Cassen, P. 1984, ApJ, 286, 529

Whitworth, A., \& Summers, D. 1985, MNRAS, 214, 1

Winkler, K. H., \& Newman, M. J. 1980, ApJ, 236, 201

Yahil, A., Johnston, M. D., \& Burrows, A. 1987, preprint

Yorke, H. W., Bodenheimer, P., \& Laughlin, G. 1993, ApJ, 41 1, 274

Zhou, S. 1992, ApJ, 394, 204 
